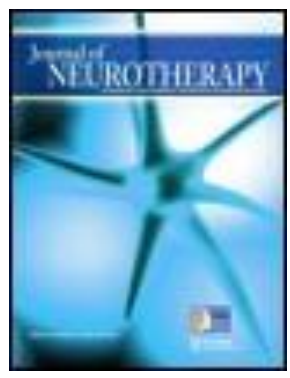

Journal of Neurotherapy: Investigations in Neuromodulation, Neurofeedback and Applied Neuroscience

\title{
Audio-Visual Entrainment Program as a Treatment for Behavior Disorders in a School Setting
}

\author{
Michael Joyce $M A^{a} \&$ Dave Siever CET ${ }^{b}$ \\ a Neurofeedback/AVE Department, "A Chance to Grow,” a special \\ needs school, Minneapolis, MN, USA \\ b Comptronic Devices, Ltd. \\ Published online: 17 Oct 2008.
}

To cite this article: Michael Joyce MA \& Dave Siever CET (2000) Audio-Visual

Entrainment Program as a Treatment for Behavior Disorders in a School Setting, Journal of Neurotherapy: Investigations in Neuromodulation, Neurofeedback and Applied

Neuroscience, $4: 2$, 9-25

To link to this article: http://dx.doi.org/10.1300/J184v04n02_04

\section{PLEASE SCROLL DOWN FOR ARTICLE}

(c) International Society for Neurofeedback and Research (ISNR), all rights reserved. This article (the "Article") may be accessed online from ISNR at no charge. The Article may be viewed online, stored in electronic or physical form, or archived for research, teaching, and private study purposes. The Article may be archived in public libraries or university libraries at the direction of said public library or university library. Any other reproduction of the Article for redistribution, sale, resale, loan, sublicensing, systematic supply, or other distribution, including both physical and electronic reproduction for such purposes, is expressly forbidden. Preparing or reproducing derivative works of this article is expressly forbidden. ISNR makes no representation or warranty as to the accuracy or completeness of any content in the Article. From 1995 to 2013 the Journal of Neurotherapy was the official publication of ISNR (www. Isnr.org); on April 27, 2016 ISNR acquired the journal from Taylor \& Francis Group, LLC. In 2014, ISNR established its official open-access journal NeuroRegulation (ISSN: 2373-0587; www. neuroregulation.org).

THIS OPEN-ACCESS CONTENT MADE POSSIBLE BY THESE GENEROUS SPONSORS

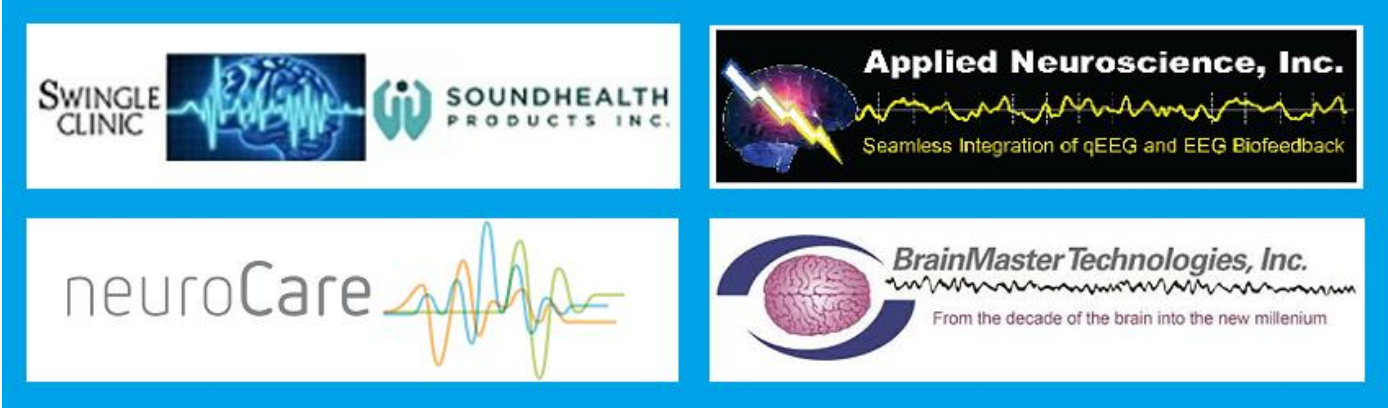




\title{
Audio-Visual Entrainment Program as a Treatment for Behavior Disorders in a School Setting
}

\author{
Michael Joyce, MA \\ Dave Siever, CET
}

\begin{abstract}
Introduction. It has been suggested that the behavioral manifestations of attention deficit hyperactivity disorder (ADHD) are secondary to neurological abnormalities and are characterized as low brain wave disorders. ADHD children produce higher amounts of theta $(5-7 \mathrm{~Hz})$ and less beta $(13-21 \mathrm{~Hz})$ brain wave activity than normals. Many researchers are testing the therapeutic effectiveness of AudioVisual Entrainment (AVE) as a treatment for a variety of low arousal
\end{abstract}

Michael Joyce, MA, has been working with special needs children since 1974 . He began working with audio-visual entrainment (AVE) in 1992. Mr. Joyce is currently the Director of the Neurofeedback/AVE Department at "A Chance to Grow," a special needs school in Minneapolis, MN.

Dave Siever, CET, has been involved in designing and manufacturing TENS and biofeedback products since 1981. He began the design of audio-visual entrainment products in 1984. David Siever, a co-author of this study, is the President of Comptronic Devices, Ltd., and Mind Alive in Edmonton, Alberta, Canada, the manufacturer and distributor of the AVS device reported in this study. As such, he has substantial commercial interest in the study.

Address correspondence to: Dave Siever, Comptronic Devices Limited, 9008 51st Avenue, Edmonton, Alberta, Canada T6E 5X4 (E-mail: dave@comptronic.com). 
brain disorders. AVE is the repetitive and intermittent presentation of light and sound. AVE affects electroencephalographic (EEG) output in that brain wave output can be suppressed or enhanced at specific frequencies.

Procedure. Thirty-four elementary students from two different schools were given AVE over the course of seven weeks. Participants were given the Test of Variables of Attention (TOVA) before and after participation. A second group of eight participants were in a special reading (SPALDING) class. All of the students in this class received the Standardized Test for the Assessment of Reading (STAR) and were compared with a control group, $\mathrm{n}=12$.

Results. Overall inattention, impulsivity and variability as rated by the TOVA improved significantly. The eight students from the SPALDING reading program who received AVE improved their reading scores more than their classmates who served as controls. The results included normalization as recorded on the TOVA, substantial improvements in reading as recorded on the STAR, and improvements in general behavior as noted by teachers and parents.

Discussion. The data suggests AVE was a useful experience for the participants. Parents and teachers reported the children were calmer and could focus better. The results met or exceeded our expectations.

KEYWORDS. Audio-visual entrainment (AVE), audio-visual stimulation (AVS), attention deficit disorder (ADD), learning difficulties (LD), tests of variables of attention (TOVA), academic performance

\section{INTRODUCTION}

It has been suggested that the behavioral manifestations of attention deficit hyperactivity disorder (ADHD) are secondary to neurological abnormalities (Zametkin et al., 1990; Zametkin et al., 1993; Mann, Lubar, Zimmerman, Miller, \& Muenchen 1992). Electroencephalographic (EEG) recordings reveal that ADHD children produce more theta $(4-7 \mathrm{~Hz})$ brainwave activity in the frontal and central cortical regions of the brain than normals (Mann et al.). This brainwave pattern is usually associated with drowsiness and low arousal. ADHD children also produce less beta $1(13-21 \mathrm{~Hz})$ brainwave activity than normals (Mann et al.). Dominant beta brainwave activity is associated with higher levels of arousal. These abnormalities in levels of theta and beta activity have been interpreted as evidence supporting the theory that ADHD is a disorder of low levels of arousal. Studies using positron emissions tomo- 
graphy confirm that ADHD is also characterized by reduced cerebral blood flow and lower levels of a glucose metabolism (Zametkin et al., 1990; Zametkin et al., 1993). To compensate for this under arousal, stimulant medication is often prescribed as a treatment for ADHD, and appears to have a calming affect on children that otherwise cannot focus or remain still (Zentall, 1975).

Changing the cerebral electrical activity associated with ADHD has improved ADHD children's symptoms (Lubar, 1991; Utter, 1996; Russell, 1997). As an alternative treatment approach to ADHD, neurofeedback has been used as a means to increasing cerebral activity (Lubar, 1991; Lubar \& Shouse, 1977; Lubar \& Deering, 1981; Lubar \& Lubar, 1984; Tansey, 1990). Differential neurofeedback in which ADHD children train with beta (15-19 $\mathrm{Hz}$ ) on site C3 and train with SMR (12-15 Hz) on site C4 based on the 10-20 electrode placement standard (Othmer, 1998), has become increasingly popular in recent years.

Many researchers are testing the therapeutic effectiveness of Audio-Visual Entrainment (AVE) as a treatment for a variety of low arousal brain disorders. AVE is the repetitive and intermittent presentation of light and sound. AVE affects electroencephalographic (EEG) output (Toman,1940; Walter, 1956; Barlow, 1960; Inouye, Sumitsuji, \& Matsumoto, 1979; Kinney, McKay, Mensch, \& Luria, 1972; Nogawa, Katayama, Tabata, Ohshio, \& Kawahara, 1976; Lesser, Luders, Klem, \& Dinner, 1986; Frederick, Lubar, Rasey, Brim, \& Blackburn, 1999). It is purported to alter perception and consciousness (Glicksohn,1986; Richardson \& McAndrew, 1990; Freedman \& Marks, 1965). AVE has been used to improve grade-point average in college students (Budzynski, Jordy, Budzynski, Tang, \& Claypoole, 1999). AVE can induce relaxation (Manns, Miralles \& Adrian, 1981; Thomas \& Siever, 1989; Brauchli, 1993; Morse \& Chow, 1993), hypnotic states (Kroger \& Schneider, 1959; Sadove, 1963; Lewerenz, 1963; Margolis, 1966) and dissociation (Leonard, Telch, \& Harrington, 1999; Leonard \& Telch, 1998). AVE has been used to reduce chronic pain (Boersma \& Gagnon, 1992), to treat migraine headache (Anderson, 1989), and to treat depression (Kumano, Horie, Shidara, Kuboki, Suematsu, \& Yasushi, 1996). AVE has produced significant reductions in anxiety (Leonard \& Telch, 1998). AVE has been used as a treatment for low-arousal brain disorders such as pre-menstrual syndrome (PMS) (Noton, 1996), chronic fatigue syndrome (Trudeau, 1999), fibromyalgia (Berg \& Siever, 2000) and seasonal affective disorder (Berg \& Siever, 2000). Low arousal brain disorders are disorders that can be characterized by abnormal EEG patterns. ADHD is one of these disorders (Noton,1996; Carter \& Russell, 1993). It has been suggested that many clinicians are using AVE informally for ADHD with anecdotal reports of successful treatment, but with few published results (Noton,1996). Carter \& Russell (1993) conducted a pilot study using AVE to treat learning and behavioral disorders. The results were 
improvements in IQ scores and behavior. Russell (1997) states that AVE achieves the same results as EEG biofeedback but at less cost and in less time.

The purpose of these studies was to expand on the ADHD research of Carter and Russell (1993), and to further determine the effectiveness of AVE treatment in reducing the symptoms associated with learning disorders such as impaired reading. Improvements in pre- and post-scores on a continuous performance task (TOVA) and on a standardized reading test (STAR) were expected.

\section{METHOD}

\section{Participants}

Thirty-four elementary students (thirteen females) participated in the studies. The mean age of the participants was $9.30 \pm 1.55$ years. Fourteen students (seven females) were from a Catholic school in the same rural community. All of the Catholic school students participated in the ADHD part of the study. Twenty students (six females) were from a public school. All twenty students were on the SPALDING reading program. Eight (four girls) of the twenty students in the SPALDING reading program with the poorest marks were selected for the treatment group and the remaining twelve students in the class (two girls) served as controls. The pre-post TOVA data from the treatment were also used in the ADHD study along with the parochial students. Students with a history of distractibility and of distracting others were selected. The selection criterion was teacher referral at the public school and parent referral at the parochial school. None of the students had a history of epilepsy or seizure. Two students from the parochial school had no academic or attention problems. These two students were interested in increasing their already adequate academic performance.

Specific diagnoses are as follows: no diagnosis by a medical physician, but a history of distractibility and misbehavior, 12; unspecified learning disorder, 10; suspected ADHD, 8; physician diagnosed ADHD, 6; emotional/ behavior disorder, 2; and one each of anxiety, allergies, fetal alcohol syndrome, and suspected depression. The sample size doesn't match the number of diagnoses because many students had multiple diagnoses.

\section{Apparatus}

The AVE device used was the DAVID Paradise XL (manufactured by Comptronic Devices Limited, Edmonton, Alberta, Canada). The eyeglasses for the DAVID Paradise XL are field independent, in that they are able to independently stimulate the individual left and right visual fields of each eye 
if selected. In this study, independent field stimulation was chosen. The parameters of the stimulation "session" were as shown in Figure 1.

The frequency and intensity may be programmed to stimulate the left and right fields of vision independently. The DAVID Paradise XL was attached to a multi-user amplifier, which enabled ten students to participate simultaneously. Each student had his/her own station, which consisted of a set of headphones and an eye set. The students could control both the audio volume and the light intensity. The students preferred brighter intensities between approximately 400 and 600 lux (full spectrum) measured approximately 0.3 inches from the eye set screen (approximating their average eye distance from the screen).

Because photic induced seizures involving those aged from five to twentyfour years of 1/4000 has been reported by Newmark and Penry (1979) and Jeavons, Bishop, and Harding (1986), care must be taken when delivering photic stimulation to children. Physiological photic stimulators generally used to induce seizures employ Xenon strobe lights that reach maximum brightness within 50 micro-seconds at intensities of 10,000-300,000 lux. Carterette and Symmes (1952) first reported that red flicker provoked an increased photo-convulsive response (PCR) relative to other wavelengths. Since then, this finding has been reported by Bickford (1953); Marshall, Walker, and Livingston (1953); Pantelakis, Bower, and Jones (1962); Kojima, Suguro, and Miyamoto (1963); Brausch and Ferguson (1965); Harley, Baird, and Freedman (1967); Takahashi and Tsukahara (1972a, 1973). It has also been reported by Carterette and Symmes (1952); Brausch and Ferguson (1965); Buskirk, Casby, Passouant, and Schwab (1952); Marshall et al. (1953); Bickford (1954); Asano and Umezaki (1965); Maruyama and Maruyama

FIGURE 1. Left and right hemisphere flash rate for the experiment specific AVE session.

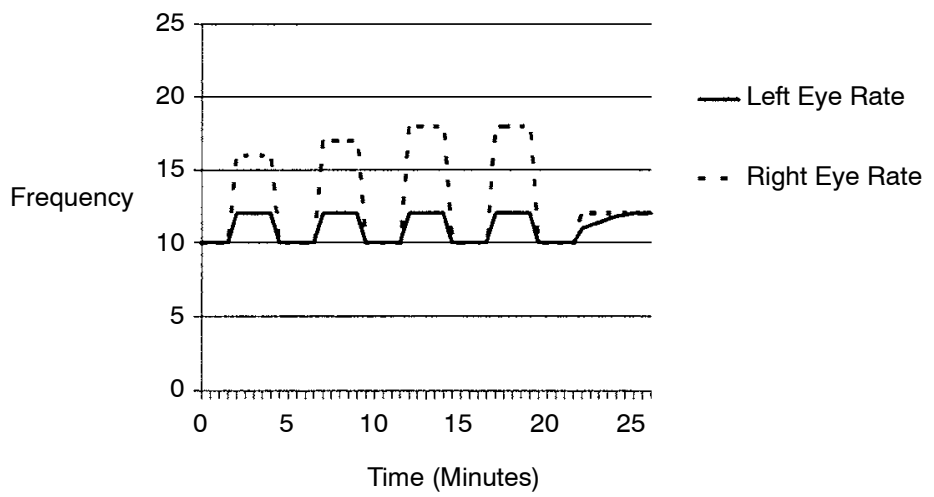


(1968); Takahashi and Tsukahara (1972b) and Harley et al. (1967) that red removing eyeglasses or contact lenses afforded clinical relief to patients with photogenic epilepsy. Kasteleijn-Nolst Trenite (1989) found that in one hundred PCR participants, eighty-one showed sensitivity with eyes closed while sixty-six were sensitive with their eyes open. Harding and Jeavons (1994) found that peak PCR sensitivity occurs from fifteen to twenty flashes per second. Takahashi and Tsukahara (1976) measured IPS induced PCRs under controlled lighting conditions. They observed that PCRs were most frequently induced with red light stimulation from $15-20 \mathrm{~Hz}$ and that it was superior in producing PCRs than stroboscopic (white) light. In all fourteen cases generalized PCRs of sharp and wave and spike and wave complex were induced. They also found that $20 \mathrm{~cd} / \mathrm{m}^{2}$ were inhibited by blue light of 1.9 $\mathrm{cd} / \mathrm{m}^{2}$. All of these studies used a brief, intense flash pulse. Ruuskanen-Uoti and Salmi (1994) reported on a person who developed seizures while using a "light and sound" machine utilizing square wave stimulation delivered by red light emitting diodes (LEDs).

Brief, intense flashes produce harmonic activity in the brain (Van der Tweel \& Verduyn, 1965); whereas, sine wave stimulation produces a sine-like response (insignificant harmonic activity). Van der Tweel and Verduyn (1965), Townsend (1973), Donker, Njio, Storm Van Leeuwen and Wieneke (1978) and Regan (1965) all agree that sine-wave modulated light eliminates the problem of light intensity from a Xenon strobe increasing with frequency and the harmonics generated within the neo-cortex at frequency multiples much higher than the fundamental at times. It has been our observations that square wave LED flashing at $7 \mathrm{~Hz}$ can produce strong harmonics between 20 and 40 $\mathrm{Hz}$. Of these sine wave stimulation studies, the concern of inducing seizures is completely omitted from the study. In the raw EEGs shown in the studies, there are neither signs of epileptiform activity nor any discussion about it.

To address the concerns of eliciting a photic induced seizure, the eye sets used had a slowed turn-on and -off time of about $15 \mathrm{msec}$. The light emitted from the eye sets was white light produced by incandescent bulbs over which was a translucent plastic sheet that was tinted a light blue.

The students were given pulsing isochoric tones at a frequency of $170 \mathrm{~Hz}$, through a set of headphones. The students could control the volume of the sounds heard through the headphones from "off" to approximately to $70 \mathrm{db}$ at their own station, using an auditory C-weighting measurement. All of the students preferred a sound volume in the range of 60 to $70 \mathrm{db}$. The students could not control the program or "session" which they were presented.

\section{Procedure}

Before treatment began, the participants were administered the Test of Variables of Attention (TOVA) (Greenburg 1987, Leark, Dupuy, Greenberg, 
Corman \& Kindschi, 1996). The TOVA is a continuous performance test used to measure impulsivity and inattention. The TOVA is used as an assessment tool in the treatment of attentional disorders (Lubar, Swartwood, Swartwood, \& O'Donnell, 1995). It has been shown to be very reliable in longterm test-retest reliability (Llorente, Amado, Voigt, Beretta, Fraley, \& Heird, in press). The eight participants in the reading class were also administered the Standardized Test for the Assessment of Reading (STAR). The STAR is a computer-adaptive, norm-referenced reading test. All pre-assessments were completed within ten days prior to the onset of the AVE sessions.

The AVE sessions took place on school days for a seven-week period beginning on September 29, 1997. The public school had sessions with ten students at 8:20 a.m. and another ten students at 12:20 p.m. The parochial school had sessions at 1:30 p.m. with eight students, and at 2:15 p.m. with another six students. There were a total of thirty-five sessions provided. The students experienced a range from twenty-eight to thirty-three sessions with a mean of thirty-one sessions. No students dropped out of the study. The sessions were provided in a quiet, dimly lit room. Before each day's session, each student consumed a glass of water. Each student got into a comfortable reclining position on a mat for the AVE session. When the eye sets and headphones were in position, the session would begin.

It was soon found necessary to provide modified audio entrainment by providing an auditory stimulus in addition to that of the AVE device in order to engage some of the students. It was decided to use environmental sounds and instrumental music. These tapes were believed to capture the students' attention. An EEG evaluation to determine if the tapes affected brainwave activity was not done. Four tapes matching these criteria were selected. The environmental sounds included birds, crickets, and rain. The instrumental music used strings and percussion, and could be described as background music. None of these tapes was marketed for relaxation or other purposes. A single tape was randomly selected for each day's session. All students received stimulation from these tapes.

A total of thirty-five AVE sessions were offered. The first eight sessions were twenty minutes long, and consisted of a low alpha (7 to $9 \mathrm{~Hz}$ ) protocol. These protocols are generally used to induce relaxation. As discussed previously, children with ADHD have EEG patterns dominated by theta. Therefore it would seem counter intuitive to induce a similar EEG pattern in ADHD children. However, regardless of the diagnoses, many students experience anxiety for various reasons and may not know how to consciously relax. It was deemed necessary to have the students relax with this stimulation to help enhance the effectiveness of the second protocol. After the initial mu protocol, the participants began the remaining sessions of a twenty-twominute SMR-beta program (see Figure 1). The frequencies selected were 
based on Carter and Russell's work (1993) and on Othmer's (1998) neurofeedback protocols for treating ADD.

\section{RESULTS}

Pre- and post-average results were calculated for the entire group of students. Table 1 lists the pre- and post-treatment group standard score means on the TOVA scales of inattention, impulsivity, reaction time, and variability. Standard deviation and significance are also presented based on the statistical ANOVA procedures described in the TOVA manual. Pre- and post-TOVA results were calculated separately for the catholic school students and the public school students. The public school students' pre-AVE TOVA scores were higher than the catholic school students' pre-AVE scores. The post-AVE scores were similar for both. Two separate groups' TOVA scores for attention, impulsivity, reaction time and variability are presented in Tables 2 and 3 . Of the fourteen Catholic students, three students had pre-AVE TOVA results

TABLE 1. Mean TOVA Standard Scores for Total Groupa

\begin{tabular}{lccc}
\hline Scale & Pre-Score & Post-Score & Difference \\
\hline Inattention & 80 & 100 & $20^{\star \star}$ \\
Impulsivity & 88 & 109 & $21^{\star}$ \\
Reaction Time & 80 & 94 & 14 \\
Variability & 80 & 103 & $23^{\star \star}$ \\
\hline $\mathrm{n}=34,{ }^{\star} \mathrm{p}<.05,{ }^{* \star \mathrm{p}}=.01$ & & & \\
\hline
\end{tabular}

TABLE 2. Mean TOVA Standard Scores for Public Schools Group ${ }^{a}$

\begin{tabular}{lccc}
\hline Sub-Scale & Pre & Post & Difference \\
\hline Inattention & 92 & 102 & 10 \\
Impulsivity & 99 & 112 & 13 \\
Reaction Time & 85 & 93 & 8 \\
Variability & 87 & 102 & $15^{\star}$ \\
\hline $\mathrm{n}=20,{ }^{*} \mathrm{p}=.05$ & & & \\
\hline
\end{tabular}


TABLE 3. Mean TOVA Standard Scores for Catholic School Group ${ }^{a}$

\begin{tabular}{lccc}
\hline Sub-Scale & Pre & Post & Difference \\
\hline Inattention & 57 & 95 & $38^{\star \star}$ \\
Impulsivity & 73 & 107 & $34^{\star \star}$ \\
Reaction Time & 68 & 94 & $26^{\star}$ \\
Variability & 68 & 103 & $35^{\star \star}$ \\
\hline $\mathrm{n}=14,{ }^{\star} \mathrm{p}<.01,{ }^{\star \star} \mathrm{p}<.001$ & & & \\
\hline
\end{tabular}

that were invalid due to extremely deviant results. These students' scores were more than 3 standard deviations below the norm before AVE. After AVE all were within normal range. The mean data on these students is presented in Table 3.

Four of the twenty students from the public school had invalid pre- and post-TOVA scores. These were due to excessive commission errors or anticipatory results to which the TOVA will automatically not process the results. These students' data were not included for the group analysis.

There were twelve students in the special reading class who did not participate in the AVE program. These students did complete the STAR and their STAR results were compared to the results of the students who participated in the AVE program. Results are presented in Figures 2 through 5.

Grade equivalent (Figure 2) ranges from 0.0 to $13.0+$ and represents a student's reading level relative to a norming sample. Instructional reading level (Figure 3) is the level at which the student can comprehend written material with assistance, at $80 \%$ proficiency. Normal curve equivalents (Figure 4) are similar to percentile ranks, but are based on an equal interval scale. Comparisons of these measures between pre and post treatment are shown in Figure 5.

After the eight alpha $(7.8 \mathrm{~Hz})$ sessions nearly all of the students had demonstrated some semblance of deep relaxation as evidenced by their rhythmic and generally deeper diaphragmatic breathing. In addition, there were teacher and parent reports of students being mellower, centered, focused and "easier going." Most of the students concurred with these impressions by reporting that they experienced more relaxation with each daily session.

The anecdotal reports at the end of the alpha-beta protocol were positive and encouraging. There were reports of shy and anxious students "coming out of their shell," and actively engaging and participating in classroom activities. In general, students also gained assertiveness and began "showing 
FIGURE 2. Grade equivalence as measure by the STAR comparing the AVE group from the non-AVE group.

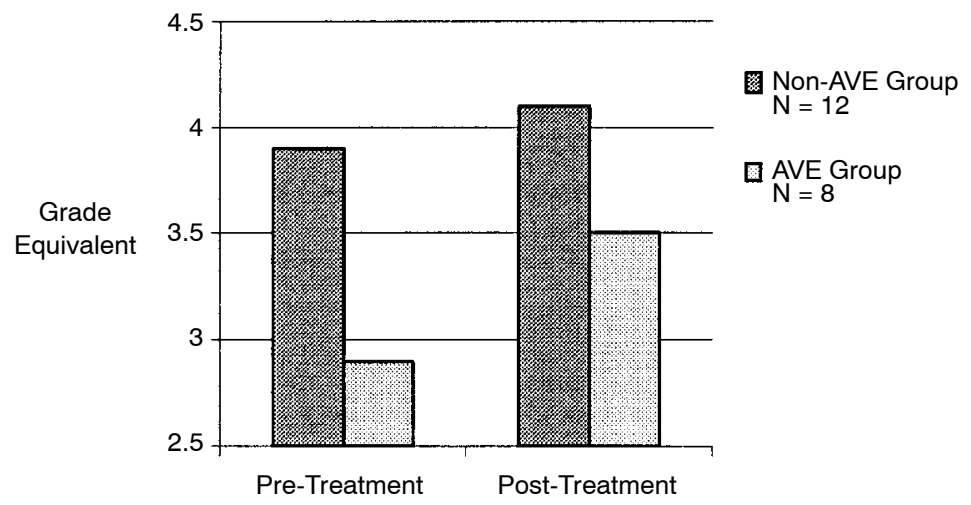

FIGURE 3. Percentile rank on the STAR compared between the AVE group and the non-AVE group.

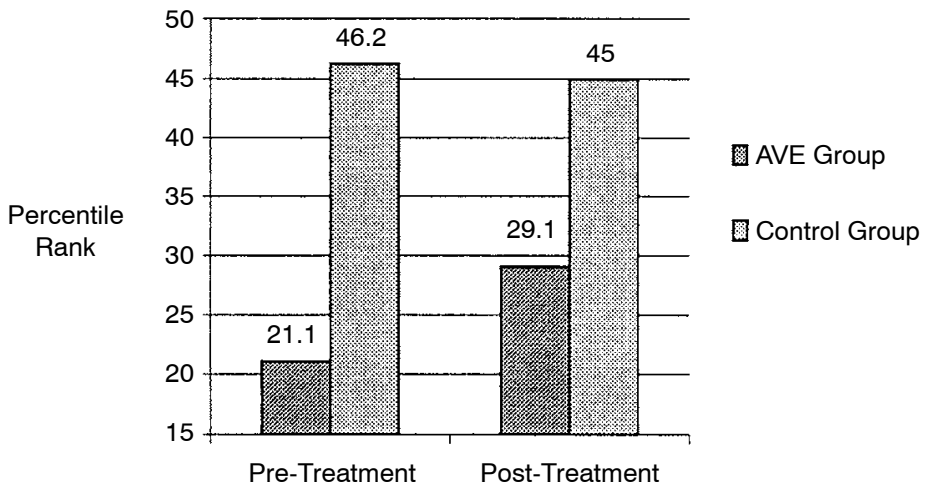

a personality," whereas previously they were generally passive and not responsive. There were also anecdotal reports of improvements in spelling, reading, fluency, self-esteem, and happiness.

Two students have reduced their dosage of Ritalin by $40 \mathrm{mg}$ each. Their dosages were reduced to $5 \mathrm{mg}$, twice per day and $10 \mathrm{mg}$, twice per day. Parents and teachers report that these two children are doing "fantastic." Other students on the program who were candidates for medication are no longer candidates for medication. 
FIGURE 4. Normal curve equivalents on the STAR comparing the AVE and non-AVE groups.

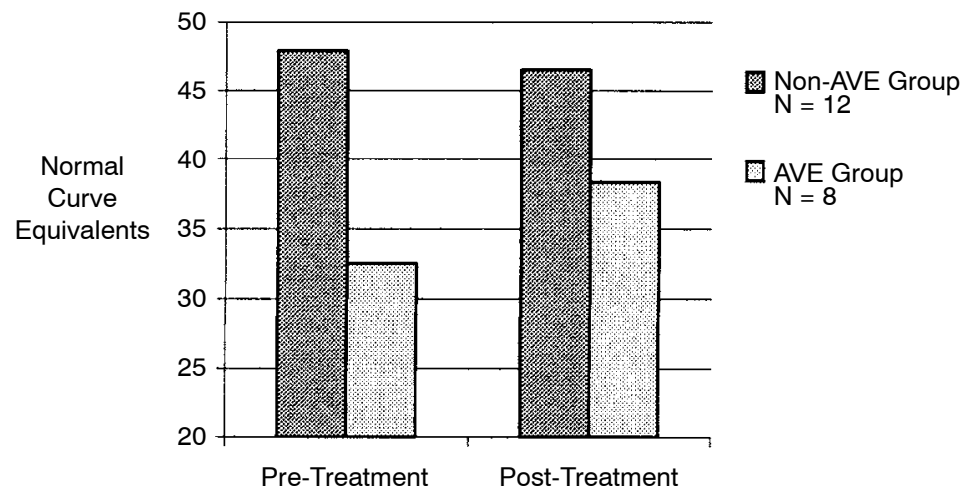

FIGURE 5. Pre- and post-AVE STAR results comparing the AVE and the non-AVE groups.

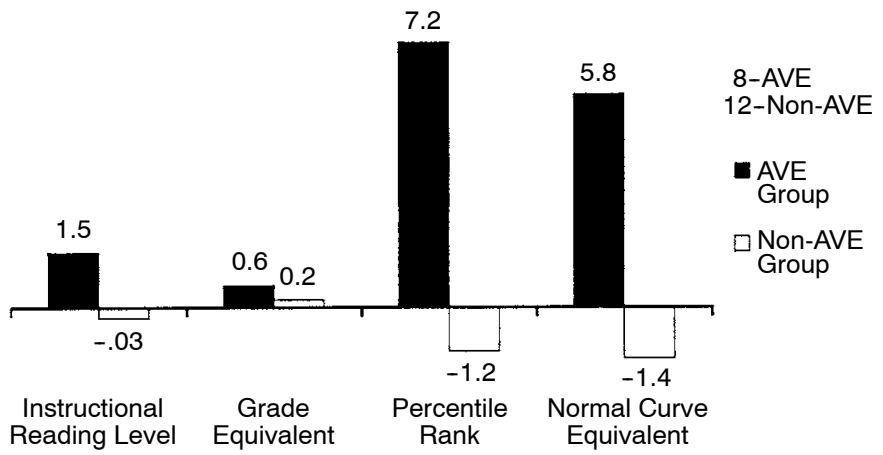

\section{DISCUSSION}

As the data suggests, AVE was a useful experience for the participants. The results met or exceeded our expectations. Most of the students had noticeable improvements as confirmed by teachers and parents. Participants whose scores were discrepant consistently did not "get into" the sessions. They were occasionally agitated but reported that they enjoyed the experience. It was felt that these students could have benefited further with more alpha/theta sessions, or with a combination of neurofeedback and AVE. 
The participants in the reading program were only a portion of the class. All remaining students were in the elementary K-4 $(n=512)$ participating in the self-esteem class at the same time daily. This set up had provided us with a control situation. The non-AVE students were given comparable therapeutic attention to the AVE students and the AVE students improved more than their peers. AVE seemed to be a better concurrent treatment than a self-esteem program for the reading program.

Several conclusions can be made. AVE proved to be an effective tool for treating learning disabilities of the type described and as a treatment for behavior disorders. This investigation suggests new possibilities for hemisphere specific stimulation. It appears to follow the model implemented by Othmer, Kaiser and Sterman (1998) where left hemisphere beta and right hemisphere SMR neurofeedback training are used successfully. This inquiry has also provided a replicable, cost-effective model, which school systems can adopt.

There are several good reasons AVE technology should be available in school settings: most communities or parents that need neuro-technologies such as AVE or neurofeedback the most, can afford it the least; travel time is minimized; immediate and ongoing feedback and random on-site observations are more easily obtained; and medication intakes can be reduced.

One of the most exciting implications is that a whole host of seemingly unrelated disorders may all be dysregulation of arousal, which may be defined as an over-activation or under-activation of the sympathetic or parasympathetic systems. Arousal dysregulation disorders may include depression, anxiety, phobias, panic, dysthymia, obsessive-compulsive disorder, post-traumatic stress disorder, hypertension, closed head injury, narcolepsy, alcoholism, Tourette's syndrome, and ADHD (Othmer, in press). All these disorders may be traced to proxysmal events in the brain spikes, slow brainwaves, irregularities, seizures and brain storms of greater or lesser magnitude (Othmer, in press). As Othmer (in press) states, "These disorders can be significantly modified by altering and bringing more order or coherence to brainwave activity and giving individuals more power of self-regulation of brain states through the use of AVE and/or EEG feedback techniques."

The authors speculate that AVE and neurofeedback may work in part not by affecting specific frequencies, but by breaking up rigidities and increasing the brain's functional flexibility. Flexibility may be defined as the ability to move quickly between various coherent brain states and frequencies; between alert and diffuse attention, or between rest and reaction. Mental flexibility is the hallmark of good mental health and peak performance. AVE may be seen as exercising the brain, making it more capable of responding to presented stimuli and taking advantage of presented stimuli.

It appears that in many cases, children referred for ADHD or LD are 
students experiencing ongoing anxiety and tension that interfere in all aspects of their life. It has been the senior author's experience that once this anxiety is addressed with either AVE or EEG biofeedback, the symptoms that warranted the referral for diagnosis are diminished or eliminated. Misdiagnoses cannot be ruled out either.

Though appearing cost-effective as compared to neurotherapy, AVE will certainly not solve all behavior or learning issues in a school setting. Schools often make the assumption that their students are free of stress, family problems, are properly nourished, properly hydrated, and are free of allergies. This is not always the case.

\section{REFERENCES}

Anderson, D. J. (1989). The treatment of migraine with variable frequency photostimulation. Headache, 29, 154-155.

Asano, T., \& Umezaki. (1965). Photogenic epilepsy-presentation of case (in Japanese). Clinical Neurology, 5, 519-523.

Barlow, J. S. (1960). Rhythmic activity induced by photic stimulation in relation to intrinsical activity of the brain in man. Electroencephalography and Clinical Neurophysiology, 12, 317-326.

Berg, K. \& Siever, D. (2000a). Audio visual entrainment as a treatment modality for seasonal affective disorder. Presentation at AAPB 31st Annual Meeting. Denver, CO.

Berg, K. \& Siever, D. (2000b). The treatment of chronic fatigue syndrome with audio visual entrainment. Presentation at AAPB 31st Annual Meeting. Denver, CO.

Bickford, R., Daly, D. \& Keith, H.M. (1953). Convulsive effects of light stimulation in children. American Journal of Diseases of Children, 86, 170-183.

Bickford, R. (1954) Sensory precipitation of seizure. Journal of Michigan Medical Society, 53, 1018-1020.

Boersma, F. J. \& Gagnon, C. (1992). The use of repetitive audio-visual entrainment in the management of chronic pain. Medical Hypnoanalysis Journal, 7, 80-97.

Brauchli, P. (1993). Vergleichsuntersuchung der Psychophysiologischen Entspannungseffekte Einer Optisch-akustischen Mind Machine mit Einer Entspannungsmusik [A Comparative Study of the Psychophysiological Relaxation Effects Induced by an Optical-acoustic Mind Machine and Relaxing Music]. Zeitschrift für experimentelle un angewandte Psychologie, 90 (2), 179-193.

Brausch, C. \& Ferguson, J. (1965). Color as a factor in light-sensitive epilepsy. Neurology, 15, 154-164.

Budzynski, T., Jordy, J., Budzynski, H., Tang, H., \& Claypoole, K. (1999). Academic performance enhancement with photic stimulation and EDR feedback. Journal of Neurotherapy, 3(3), 11-21.

Buskirk, C., Casby, J., Passouant, P. \& Schwab, R. (1952). The effect of different modalities of light on the activation of the EEG. EEG and Clinical Neurophysiology, 4, 244-245.

Carter, J. L., \& Russell, H. L. (1993). A pilot investigation of auditory and visual entrainment of brain wave activity in learning disabled boys. Texas Researcher, Journal of the Texas Center for Educational Research, 4, 65-73. 
Carterette, E. C. \& Symmes, D. (1952). Symposium: Photo-Metrazol activation of electroencephalogram; Color as experimental variable in photic stimulation. Electrenceph. Clin. Neurophysiology. 4, 289-296.

Donker, D., Njio, L., Storm Van Leeuwen, W., \& Wieneke, G. (1978). Interhemispheric relationships of responses to sine wave modulated light in normal subjects and patients. EEG and Clinical Neurophysiology, 44, 479-489.

Frederick, J., Lubar, J. L., Rasey, H., Brim, S., \& Blackburn, J. (1999). Effects of 18.5 $\mathrm{Hz}$ auditory and visual stimulation on EEG amplitude at the vertex. Journal of Neurotherapy, 3(3), 23-27.

Freedman, S., \& Marks, P. (1965). Visual imagery produced by rhythmic photic stimulation: personality correlates and phenomenology. British Journal of Psychology, $56(1), 95-112$.

Glicksohn, J. (1986). Photic driving and altered states of consciousness: An exploratory study. Imagination, Cognition and Personality, 6, 167-182.

Greenburg, L. M. (1987). An objective measure of methylphenidate: clinical use of the MCA. Psychopharmacology Bulletin, 23, 279-282.

Harley, R., Baird, H. \& Freedman, R. (1967). Self-induced photogenic epilepsy. Report of four cases. Arch Opthal. 78, 730-737

Harding, F. A. \& Jeavons, P. M. (1994). Photosensitive epilepsy, New Edition, Suffolk: Lavenham Press, Ltd.

Inouye, T., Sumitsuji, N., \& Matsumoto, K. (1979). EEG changes induced by light stimuli modulated with the subject's alpha rhythm. Electroencephalography and Clinical Neurophysiology, 49, 135-142.

Jeavons, P. M., Bishop, A. \& Harding, G. F. A. (1986). The prognosis of photosensitivity. Epliepsia, 27(5), 569-575.

Kasteleijn-Nolst Trenite, D., (1989) Photosensitivity in epilepsy: Electrophysiological and clinical correlates. Acta Neurologica Scandinavica. Supplementum; 125

Kinney, J., McKay, C., Mensch, A., \& Luria, S., (1972). Visual evoked responses elicited by rapid stimulation. EEG and Clinical Neurophysiology, 34, 7-13.

Kojima, K., Suguro, T., \& Miyamoto, K., (1963). Television epilepsy (in Japanese) Journal of Pediatric Practices, 26, 1377-1381.

Kroger, W. S. \& Schneider, S. A. (1959). An electronic aid for hypnotic induction: A preliminary report. International Journal of Clinical and Experimental Hypnosis, 7, 93-98.

Kumano, H., Horie, H., Shidara, T., Kuboki, T., Suematsu, H., \& Yasushi, M. (1996). Treatment of a depressive disorder patient with EEG-driven photic stimulation. Biofeedback and Self-Regulation, 21, 323-334.

Leark, R. A., Dupuy, T. R., Greenberg, L. M., Corman, C. L., \& Kindschi, C. L. (1996). T.O.V.A., Test of Variables of Attention, Professional Manual Version 7.0, Universal Attention Disorders Inc.

Leonard, K. N., \& Telch, M. J. (1998). Dissociation and the fear response. Unpublished manuscript. Austin: The University of Texas.

Leonard, K. N., Telch, M., \& Harrington, P. (1999). Dissociation in the Laboratory: A comparison of strategies. Behavior Research and Therapy, 37, 49-61.

Lesser, R. P., Lüders, H., Klem, G., \& Dinner, D. S. (1986). Visual potentials evoked 
by light-emitting diodes mounted in goggles. Cleveland Clinic Quarterly, 52, 223-228.

Lewerenz, C. (1963). A factual report on the brain wave synchronizer. Hypnosis Quarterly, 6(4), 23.

Llorente, A., Amado, A., Voigt, R., Beretta, M., Fraley, J. \& Heird, W. (In press). Internal consistency, temporal stability, and reproducibility of individual index scores of the Test of Variables of Attention (T.O.V.A.) in children with attention deficit/hyperactivity disorder (AD/HD).

Lubar, J. F. (1991). Discourse on the development of EEG diagnostics and biofeedback for attention-deficit/hyperactivity disorder. Biofeedback and Self-Regulation, 16, 201-225.

Lubar, J. F., \& Deering, W. M. (1981). Behavioral Approaches to Neurology. New York: Academic Press.

Lubar, J. O., \& Lubar, J. F. (1984). Electroencephalographic biofeedback of SMR and beta for treatment of attention deficit disorders in a clinical setting. Biofeedback and Self-Regulation, 9, 1-23.

Lubar, J. F., \& Shouse, M. N. (1977). Use of biofeedback in the treatment of seizure disorders and hyperactivity. In B. B. Lahey \& A. E. Kadzin (Eds.), Advances in Child Clinical Psychology. New York: Plenum Press.

Lubar, J. F., Swartwood, M. O., Swartwood, J. N., \& O’Donnell, P. H. (1995). Evaluation of the effectiveness of EEG neurofeedback training for ADHD in a clinical setting as measured by changes in T.O.V.A. scores, behavioral ratings, and WISC-R performance. Biofeedback and Self-Regulation, 20, 83-99.

Mann, C. A., Lubar, J. F., Zimmerman, A. W., Miller, C. A., \& Muenchen, R. A. (1992). Quantitative analysis of EEG in boys with attention-deficit-hyperactivity disorder: Controlled study with clinical implications. Pediatric Neurology, 8, 30-36.

Manns, A., Miralles, R. \& Adrian, H. (1981). The application of audio-stimulation and electromyographic biofeedback to bruxism and myofascial pain-dysfunction syndrome. Oral Surgery, 52(3), 247-251.

Margolis, B. S. (1966). A technique for rapidly inducing hypnosis: A valuable tool for allaying fears and apprehensions about dental treatment is made practical with an electronic instrument. Cal, June, 21-24.

Marshall, C., Walker, A. E., \& Livingston, S. (1953). Photgenic epilepsy: Parameters of activation. Arch. Neurol., (69), 760-765.

Maruyama, K. \& Maruyama, H. (1968). Light sensitive epilepsy. Clinical and EEG studies on 75 cases, especially on EEG activation by television. (In Japanese). Advanced Neurological Sciences 12, 537-553.

Morse, D. R. \& Chow, E. (1993). The effect of the Relaxodont ${ }^{\mathrm{TM}}$ Brain Wave Synchronizer on endodontic anxiety: Evaluation by galvanic skin resistance, pulse rate, physical reactions, and questionnaire responses. International Journal of Psychosomatics, 40, 68-76.

Newmark, M. \& Penry, J. (1979). Photosensitivity and Epilepsy: A Review. New York: Raven Press.

Nogawa, T., Katayama, K., Tabata, Y., Ohshio, T., \& Kawahara, T. (1976). Changes 
in amplitude of the EEG induced by a photic stimulus. Electroencephalography and Clinical Neurophysiology, 40, 78-88.

Noton, D. (1996). PMS, EEG, and photic stimulation. Poster presentation at the annual meeting of the Association for Applied Psychophysiology and Biofeedback.

Othmer, Z. (1998). Arousal and its role in many disorders. Unpublished manuscript.

Othmer, Z. (1998). Attention deficit disorder. EEG Spectrum Training Syllabus. Volume 3. Encino, CA: EEG Spectrum.

Othmer, Z., Kaiser, D., \& Sterman, B. (1998). Neurophysiology Mechanisms of EEG Biofeedback. EEG Spectrum Training Syllabus. Volume 5. Encino, CA: EEG Spectrum.

Pantelakis, S. N., Bower, B. D., \& Jones, H. D. (1962). Convulsions and television viewing. Brit. Med.J., 2, 633-638.

Regan, D. (1965). Some characteristics of average steady state and transient responses evoked by modulated light. EEG and Clinical Neurophysiology, 20, 238-248.

Richardson, A., \& McAndrew, F. (1990). The effects of photic stimulation and private self-consciousness on the complexity of visual imagination imagery. British Journal of Psychology, 81, 381-394.

Russell, H. L. (1997). Intellectual, auditory and photic stimulation and changes in functioning in children and adults. Biofeedback, 25(1), 16-17, 23, 24.

Ruuskanen-Uoti, H. \& Salmi, T. (1994). Epileptic seizure induced by a product marketed as a "Brainwave Synchronizer." Neurology, 44, 180.

Sadove, M. S. (1963). Hypnosis in anesthesiology. Illinois Medical Journal, 39-42.

Takahashi, T. \& Tsukahara, Y. (1972a). EEG activation by red color (in Japanese). Igaku No Ayumi, 83, 25-26.

Takahashi, T. \& Tsukahara, Y. (1972b). Inhibitory effect of blue color on seizure discharges (in Japanese). Igaku No Ayumi, 83, 81-82.

Takahashi, T. \& Tsukahara, Y. (1973). Study of visual epilepsy-red-flicker activation and clinical EEG findings (in Japanese). Clinical Neurology, 13, 697-704.

Takahashi, T. \& Tsukahara, Y. (1976). Influence of color on the photoconvulsive response. Electroencephalography and Clinical Neurophysiology, 41, 124-136.

Tansey, M. A. (1990). Righting the rhythms of reason, EEG biofeedback training as a therapeutic modality in a clinical office setting. Medical Psychotherapy, 3, 57-68.

Thomas, N. \& Siever, D. (1989). The effect of repetitive audio/visual stimulation on skeletomotor and vasomotor activity. In Waxman, D., Pedersen, D., Wilkie, I., \& Meller, P. (Eds.) Hypnosis: 4th European Congress at Oxford. London: Whurr Publishers.

Toman, J. (1940). Flicker potentials and the Alpha Rhythm in Man. Journal of Neurophysiology, 4, 51-61.

Townsend, R. (1973). A device for generation and presentation of modulated light stimuli. EEG and Clinical Neurophysiology, 34, 97-99.

Trudeau, D. L., Moore, J., Stockley, H., \& Rubin, Y. (1999). A pilot study of the effect of $18 \mathrm{~Hz}$ audio visual stimulation (AVS) on attention and concentration symptoms and on quantitative EEG (QEEG) in long term chronic fatigue (CFS). Abstract. Journal of Neurotherapy 3(4), 76. 
Utter, C. P. (1996). A controlled study of the effects of neurofeedback training on IQ and EEG patterns for ADD subjects. Unpublished manuscript. College of Wooster.

Van der Tweel, L. \& Verduyn, L. (1965). Human visual responses to sinusoidally modulated light. EEG and Clinical Neurophysiology, 18, 587-598.

Walter, W. G. (1956). Colour illusions and aberrations during stimulation by flickering light. Nature, 177, 710 .

Zametkin, A. J., Nordahl, T. E., Gross, M., King, A. C., Semple, W. E., Rumsey, J., Hamburger, S., \& Cohen, R. M. (1990). Cerebral glucose metabolism in adults with hyperactivity of childhood onset. The New England Journal of Medicine, 323, 1361-1366.

Zametkin, A. J., Liebenauer, L. L., King, A. C., Minunkas, D. V., Herscovitch, P., Yamada, E. M., \& Cohen, R. M. (1993). Brain metabolism in teenagers with attention-deficit hyperactivity disorder. Archives of General Psychiatry, 50, 333-340.

Zentall, S. (1975). Optimal stimulation as theoretical basis of hyperactivity. American Journal of Orthopsychiatry, 45, 549-562.

RECEIVED: $10 / 28 / 99$

REVISED: $03 / 31 / 00$

ACCEPTED: 04/15/00 